\title{
Sistemas combinados para el tratamiento de aguas residuales basados en tanque séptico - filtro anaerobio y humedales subsuperficiales
}

\author{
Carlos A. Madera* ${ }^{*}$ Juan P. Silva*, Miguel R. Peña** \\ * Escuela de Ingeniería de Recursos Naturales y del Ambiente (EIDENAR), Facultad de Ingeniería - \\ Universidad del Valle \\ ** Instituto CINARA - Universidad del Valle, Cali, Colombia \\ se-mail: cmadera@univalle.edu.co
}

(Recibido: Marzo 8 de 2005 - Aceptado: Diciembre 15 de 2005)

\begin{abstract}
Resumen
Colombia está afrontando una gran problemática de contaminación de sus fuentes hídricas, ya que gran parte de los ríos, cerca a los cuales se asienta más del $90 \%$ de la población, están siendo deteriorados por la descarga de aguas residuales sin tratar. Actualmente en el país la cobertura de sistemas de tratamiento alcanza solamente el $12 \%$. Existen trabajos de investigación que han planteado que los sistemas anaeróbicos complementados con sistemas naturales para el tratamiento de Aguas Residuales Domésticas (ARD) son una buena combinación para los pequeños municipios y comunidades rurales. Una evaluación del comportamiento del sistema Tanque Séptico (TS) + Filtro Anaerobio (FA) + Humedal de Flujo Subsuperficial (HFS) fue realizada en dos sitios, Ginebra y Cali en el Valle del Cauca, Colombia.
\end{abstract}

Palabras Clave: Aguas Residuales Domésticas, eficiencia de remoción, filtro anaerobio, humedal, sistema integrado, tanque séptico.

\begin{abstract}
Colombia is facing a growing contamination of its water resources, since many rivers (near which more than $90 \%$ of the total population is settled) are polluted by raw wastewater discharges. Currently, the average coverage of wastewater treatment in the country is around $12 \%$. There is some research work showing that anaerobic systems followed by natural systems are a good technological combination for small municipalities and rural areas. A performance evaluation of the system septic tank (ST) + Anaerobic filter (AF) + Sub-surface Constructed Wetland (SSW) was carried out in the towns of Ginebra and La Voragine, Valle del Cauca region, Colombia.
\end{abstract}

Keywords: Domestic wastewater, Removal efficiency,Anaerobic system, Constructed wetlands, Integrated system, Septic tank.

\section{Introducción}

Las aguas residuales domésticas y las agroindustriales contienen diversos compuestos potencialmente dañinos. La descarga de aguas residuales crudas en el ambiente acuático causa daños considerables a muchas formas de vida presentes en los ecosistemas. Igualmente, esta situación generó un riesgo potencial para la salud asociado a un gran número de enfermedades que son responsables del $80 \%$ de la morbilidad y mortalidad en los países del Tercer Mundo (CEPIS, 2000).

Feachem y Cairncross (1993) y Moscoso y León (1994) reportaron que sólo el $40 \%$ de la población total de América Latina y el Caribe contaba con 
servicio de alcantarillado. Esto representó una producción diaria de 40 millones de $\mathrm{m}^{3}$ de aguas residuales descargadas directamente en los ríos, lagos y mares. Se estimó que para el año 2000, se podría alcanzar una cobertura del $90 \%$ en alcantarillado, lo que significaría que cerca de 100 millones de $\mathrm{m}^{3}$ de aguas residuales serían vertidos a las fuentes receptoras. El panorama era incierto si se tiene en cuenta que para ese año, menos del $10 \%$ de los alcantarillados descarga a un sistema de tratamiento de aguas residuales. El CEPIS (2000) reportó que menos del $6 \%$ de la población total de América Latina tiene tratamiento de aguas residuales. En Colombia del $100 \%$ de las aguas residuales domésticas (ARD) producidas, sólo el $12 \%$ era tratada antes de descargarse en las fuentes superficiales (CEPIS, 2000).

Teniendo en cuenta la problemática mencionada, se hace necesario buscar alternativas tecnológicas sostenibles. El presente artículo analiza los resultados obtenidos en una investigación realizada en dos sistemas de tratamiento compuestos por Tanque Séptico (TS) + Filtro Anaerobio (FA) + Humedal de Flujo Subsuperficial (HFS), ubicados en las localidades de Ginebra y La Voragine, Valle del Cauca, Colombia. Esta alternativa presenta un buen desempeño en el tratamiento de aguas residuales domésticas y por tanto podría ser aplicada en comunidades medianas y pequeñas, en la perspectiva de generar proyectos sostenibles, altamente eficientes y de bajo costo.

\section{Metodología}

El presente estudio involucró la evaluación de dos sistemas de tratamiento de aguas residuales, uno a escala piloto y otro a escala real. El primero de ellos se ubica en la Estación de Investigación y Transferencia en Tratamiento y Reuso de Aguas Residuales Domésticas (EITG), del municipio de Ginebra, (Valle del Cauca). El segundo sistema es la planta de tratamiento de ARD de la comunidad rural La Vorágine, del municipio de Cali. La ubicación geográfica de ambos proyectos se muestra en la Figura 1.

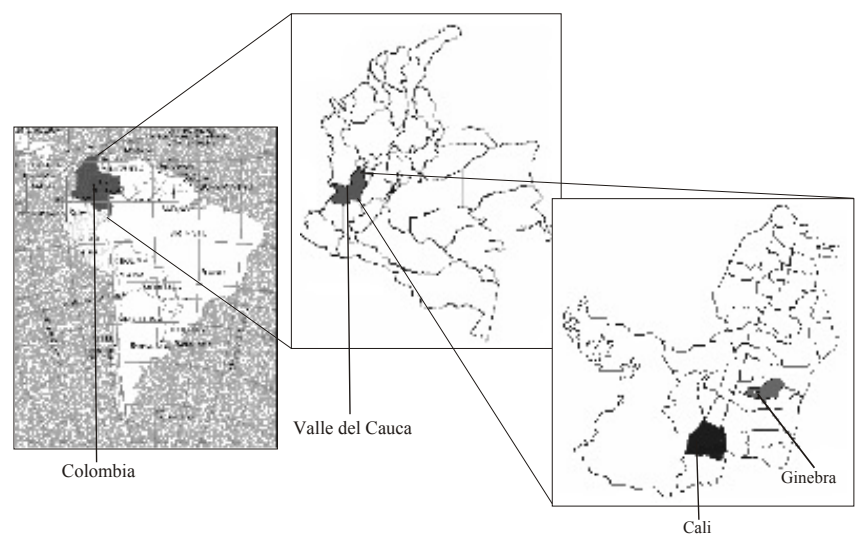

Figura 1. Ubicación de los sistemas estudiados en Cali y Ginebra.

Las unidades básicas que conforman cada sistema de tratamiento son Tanque séptico (TS) + Filtro Anaerobio de Flujo Ascendente (FA) + Humedal de Flujo Subsuperficial (HFS) sembrado con Papiros (Cyperus, sp.). La Tabla 1 presenta las características principales de las diferentes unidades y la Figura 2 muestra las unidades estudiadas.

Tabla 1. Características de las Unidades investigadas

\begin{tabular}{|c|c|c|c|c|c|c|c|c|c|}
\hline \multicolumn{2}{|c|}{ Sistema } & \multirow{2}{*}{$\begin{array}{l}\text { Escala } \\
\text { Piloto }\end{array}$} & \multirow{2}{*}{$\begin{array}{c}\text { Caudal Q } \\
\left(\mathrm{m}^{3 / \mathrm{d}}\right)\end{array}$} & \multirow{2}{*}{$\begin{array}{c}\begin{array}{c}\text { TRH } \\
\text { (dias) }\end{array} \\
0.5\end{array}$} & \multirow{2}{*}{$\begin{array}{c}\begin{array}{c}\text { Largo } \\
\text { (m) }\end{array} \\
\text { N.A }\end{array}$} & \multirow{2}{*}{$\begin{array}{c}\begin{array}{c}\text { Ancho } \\
\text { (m) }\end{array} \\
1.5\end{array}$} & \multirow{2}{*}{$\begin{array}{c}\begin{array}{c}\text { Profundidad } \\
(\mathrm{m})\end{array} \\
1.2\end{array}$} & \multirow{2}{*}{$\begin{array}{c}\text { Macrofita } \\
\text { N.A }\end{array}$} & \multirow{2}{*}{$\begin{array}{c}\begin{array}{c}\text { Diámetro } \\
\text { medio filtrante } \\
(\mathrm{m})\end{array} \\
\text { N.A }\end{array}$} \\
\hline \multirow{3}{*}{ Ginebra } & $\mathrm{TS}^{*}$ & & & & & & & & \\
\hline & $\mathrm{FA}^{*}$ & & & 0.5 & N.A & 1.5 & & N.A & 0.07 \\
\hline & HFS & & & 0.5 & 2.25 & 0.7 & & Cyperus sp & $0.07-0.10$ \\
\hline \multirow{3}{*}{ a Vorágine } & TS & & 10 & & 10 & 5. & & & N.A \\
\hline & FA & & & & 10 & 5. & & N.A. & $0.04-0.07$ \\
\hline & HFS & Real & 104 & 0.13 & 23 & 1.60 & 0.7 & Cyperus sp. & $0.04-0.07$ \\
\hline
\end{tabular}

*Unidades Circulares, Ancho = Valor del diámetro. NA: No Aplica

En el período comprendido entre noviembre de 1999 a diciembre de 2000, se realizaron dos investigaciones en el sistema de Ginebra. En ellas se estudió la influencia del caudal y por ende la variación del TRH en el funcionamiento global del sistema respecto a remoción de DQO, $\mathrm{DBO}_{5}, \mathrm{SST}$, $\mathrm{NTK}, \mathrm{N}-\mathrm{NH}_{3}, \mathrm{P}_{-} \mathrm{PO}_{4}$, coliformes fecales y huevos de helmintos. El caudal (Q, $\left.1.36 \mathrm{~m}^{3} / \mathrm{d}\right)$ aplicado fue modificado desde su valor de diseño hasta 3 veces dicho valor y para cada condición de operación se muestreó por un periodo de 30 días en estado estable. Se tomaron muestras a la entrada y salida del sistema, dos veces por semana (miércoles y sábados), a intervalos de una hora e integrando cada 6 horas durante un periodo de muestreo entre las 6:00 a.m. y 6.00 p.m. para cada día de muestreo. 


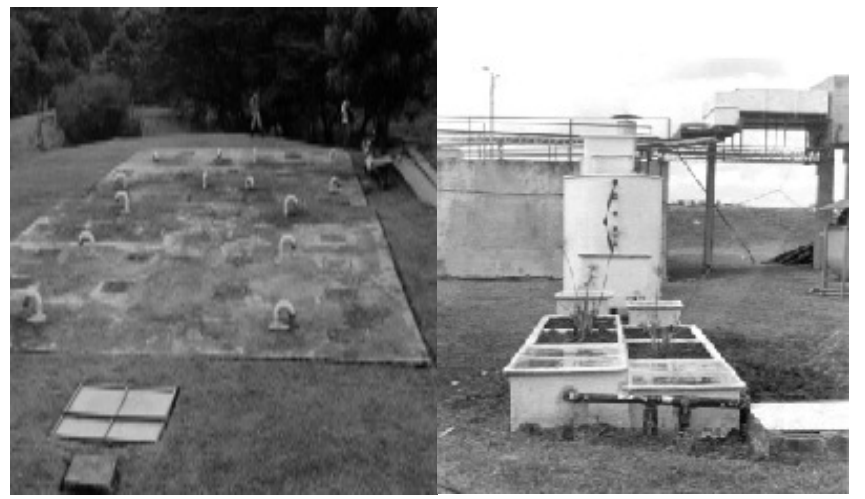

a) La Vorágine

a) Ginebra

Figura 2. Sistemas de tratamiento estudiados

En la planta de tratamiento de ARD de la comunidad de la Vorágine, se realizó una investigación en el período junio - agosto de 1998, la cual comprendió la variación del caudal aplicado así: Qd, 1.33Qd y 2Qd, $\left(Q_{d}\right.$ : Caudal de diseño del sistema, $\left.104 \mathrm{~m}^{3} / \mathrm{d}\right)$. Durante este tiempo se monitoreó en cada una de las unidades de tratamiento el caudal, temperatura, $\mathrm{pH}, \mathrm{DQO}$, DBO, SST, NTK, $\mathrm{NO}_{3}^{-}$, P-total. Apartir del estudio de la variación de caudales y de carga orgánica durante diferentes días de la semana, se determinó realizar la toma de muestras de manera compuesta los días miércoles y domingos entre las 9:00 y las 17:00 horas, integrando cada hora y tomando una alícuota proporcional al caudal medido en ese instante. La muestra colectada se almacenó en recipientes plásticos y fue preservada en una nevera portátil con gel $\pm 4^{\circ} \mathrm{C}$.

Los análisis de laboratorio se desarrollaron con base en el manual Standard Methods (APHA, 1992). El estudio del comportamiento de los sistemas de tratamiento se llevó a cabo fijando puntos de muestreo tanto a la entrada como a la salida de las diferentes unidades de tratamiento y los datos obtenidos se analizaron mediante el uso de estadística descriptiva (i.e., coeficiente variación, media aritmética y desviación estándar), todo ello con el propósito de establecer el comportamiento de las unidades respecto a las variaciones de caudal. Se analizó el comportamiento de los parámetros mencionados a través del tiempo. El paquete Excel 2000 (Microsoft Corporation) se utilizó para el análisis estadístico de los datos.

\section{Resultados y Discusión}

\section{Características del Agua Residual}

La Tabla 2 muestra la composición del agua residual cruda afluente a los dos sistemas. Para el caso de Ginebra, con base en la materia orgánica $\left(\mathrm{DBO}_{5}\right)$ el agua cruda se puede calificar como de carga media, ya que está por encima del promedio para un agua residual doméstica típica, y para La Vorágine la composición corresponde a un agua de concentración baja (Metcalf \& Eddy, 1995).

Tabla 2. Características del agua residual cruda en los sistemas estudiados

\begin{tabular}{|c|c|c|c|c|c|c|}
\hline \multirow{2}{*}{ Parámetro } & \multicolumn{3}{|c|}{ Ginebra } & \multicolumn{3}{|c|}{ La Vorágine } \\
\hline & $\mathrm{n}$ & Rango & Promedio & $\mathrm{n}$ & Rango & Promedio \\
\hline $\mathrm{T},{ }^{\circ} \mathrm{C}$ & 384 & $23-27$ & 25 & 864 & $21.6-24.0$ & 25 \\
\hline $\mathrm{pH}$ (un) & 384 & $6.6-7.1$ & N.A & 864 & $6.4-7.0$ & N.A \\
\hline SST (mg/) & 80 & $82-274$ & 140 & 96 & $60-300$ & 125 \\
\hline $\mathrm{DBO}_{5}(\mathrm{mg} / \mathrm{l})$ & 32 & $230-425$ & 356 & 24 & $136-390$ & nd \\
\hline $\mathrm{DQO}(\mathrm{mg} / \mathrm{l})$ & 80 & $254-576$ & 482 & 24 & $193-562$ & 375 \\
\hline AGV (meq/l) & 80 & $14-31$ & 22 & 24 & n.d & n.d \\
\hline NTK $(\mathrm{mg} / \mathrm{l})$ & 4 & $40.1-80.0$ & 55.3 & 96 & $3.3-69.4$ & 22.6 \\
\hline P-PO $(\mathrm{mg} / \mathrm{l})$ & 4 & $3.4-6.0$ & 4.5 & 36 & $0.3-2.5$ & nd \\
\hline $\begin{array}{l}\text { Colif. Fecales } \\
\text { (UFC/100ml) }\end{array}$ & 8 & $\begin{array}{c}1.3 \times 10^{7} \cdot 2.2 \mathrm{x} \\
10^{7}\end{array}$ & $1.8 \times 10^{7}$ & 36 & $\begin{array}{c}8.2 \times 10^{5-1.73 x} \\
10^{7}\end{array}$ & nd \\
\hline
\end{tabular}

N.A:No Aplica. n.d: No disponible.

\section{Comportamiento de la eficiencia de remoción}

La eficiencia de remoción de materia orgánica en el sistema de Ginebra no varió considerablemente a pesar de las variaciones de caudal. La Tabla 3 relaciona las eficiencias de remoción para las distintas sobrecargas de trabajo bajo las cuales operó el sistema y la Figura 3 ilustra el comportamiento general del sistema. Los resultados obtenidos en esta unidad, permiten plantear que este tipo de tecnología integrada es flexible, ya que puede trabajar bajo distintas condiciones operacionales (valores de caudal) y la capacidad de remoción expresada como $\mathrm{DQO}, \mathrm{DBO}_{5}$ y $\mathrm{SST}$ no se ve afectada considerablemente.

A partir de los datos obtenidos para el sistema de Ginebra se observa que para los valores de sobrecarga extremos, equivalentes a $1.25 \mathrm{Q}$ y $3 \mathrm{Q}$, la remoción de $\mathrm{DQO}$ sólo se reduce en promedio en un $9 \%$, mientras que para SST las eficiencias permanecen constantes. Estas altas eficiencias se obtuvieron en las dos unidades anaeróbicas de tratamiento $(\mathrm{TS}+\mathrm{FA})$. 
Tabla 3. Eficiencias de remoción para distintas cargas aplicadas al sistema de Ginebra

\begin{tabular}{cccccccc}
\hline $\begin{array}{c}\text { Carga } \\
\text { Aplicada }\end{array}$ & Q Aplicado $\left(\mathrm{m}^{3} / \mathrm{d}\right)$ & $\begin{array}{c}\mathrm{DBO} \\
\%\end{array}$ & DQO $\%$ & $\begin{array}{c}\mathrm{SST} \\
\%\end{array}$ & $\begin{array}{c}\mathrm{TKN}^{*} \\
\%\end{array}$ & $\begin{array}{c}\mathrm{P}^{\mathrm{PO}} \mathbf{4}^{*} \\
\%\end{array}$ & $\begin{array}{c}\text { Coliformes } \\
\text { Fecales } \%\end{array}$ \\
\hline $1.25 \mathrm{Q}$ & 1.7 & 82 & 77 & 87 & $\mathrm{NM}$ & 15 & 69 \\
$1.50 \mathrm{Q}$ & 2.1 & 80 & 79 & 81 & $\mathrm{NM}$ & 26 & 73 \\
$1.75 \mathrm{Q}$ & 2.4 & 71 & 82 & 88 & $\mathrm{NM}$ & 19 & 75 \\
$2.00 \mathrm{Q}$ & 2.7 & 68 & 78 & 87 & 18 & 15 & 71 \\
$3.00 \mathrm{Q}$ & 4.1 & $\mathrm{NM}$ & 68 & $\mathrm{NM}$ & 14 & 15 & 60 \\
\hline
\end{tabular}

* Eficiencias medidas solo en el Humedal. NM: Parámetros no medidos en estas condiciones de operación

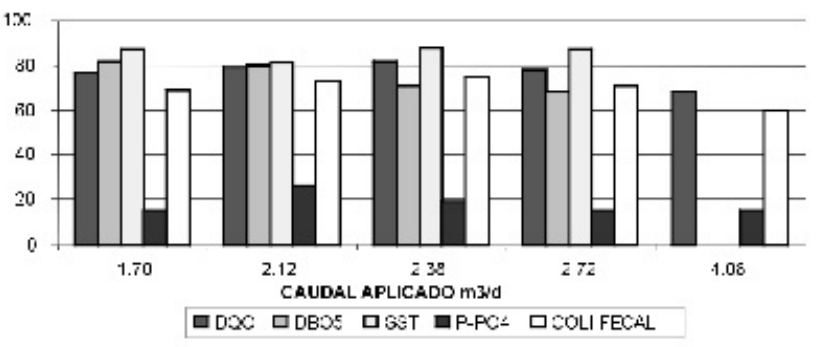

Figura 3. Variación de la eficiencia de remoción promedio a diferentes cargas hidráulicas aplicadas, Ginebra.

En el caso de La Vorágine el comportamiento fue similar al de la unidad piloto de Ginebra, ya que la eficiencia no se afectó ostensiblemente ante el aumento del caudal. En general las mayores remociones de DQO se observaron en las dos primeras unidades de tratamiento (TS y FA), situación que se mantuvo durante las tres etapas de operación del sistema. La Tabla 4 resume los resultados de remoción para los diferentes parámetros estudiados y la Figura 4 ilustra el comportamiento general del sistema respecto a la DQO.

Tabla 4. Eficiencias de remoción para distintas cargas aplicadas al sistema de La Vorágine

\begin{tabular}{cccccc}
\hline Carga Aplicada & Q Aplicado $\left(\mathrm{m}^{3} / \mathrm{d}\right)$ & $\begin{array}{c}\text { DQO } \\
\%\end{array}$ & $\begin{array}{c}\text { SST } \\
\%\end{array}$ & $\begin{array}{c}\text { TKN } \\
\%\end{array}$ & $\begin{array}{c}\text { Coliformes } \\
\text { Fecales, } \%\end{array}$ \\
\hline $1.00 \mathrm{Q}$ & 104 & 84 & 96 & 29 & 98 \\
$1.33 \mathrm{Q}$ & 138 & 83 & 93 & 21 & 90 \\
$2.00 \mathrm{Q}$ & 208 & 77 & 92 & 5 & 94 \\
\hline
\end{tabular}

La Figura 4 muestra las remociones de materia orgánica en cada unidad de tratamiento. En el caso del tanque séptico se obtuvieron eficiencias promedio de remoción de DQO para las tres condiciones de variación de caudales de $56.2 \%$ $\pm 17.7 ; 57.2 \% \pm 9.7$ y $49.3 \% \pm 10.1$, respectivamente.

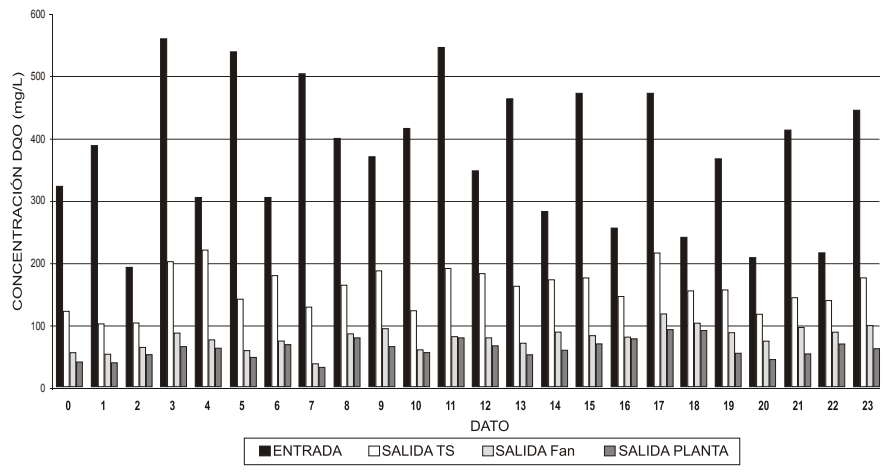

Figura 4. Variaciones de DQO para diferentes condiciones operacionales, La Vorágine

Analizando el comportamiento de los componentes del sistema, se tiene que en el FA, la eficiencia de remoción de DQO es afectada por la variación de caudal, disminuyendo la eficiencia cuando este parámetro se incrementa, situación que puede estar asociada a menor tiempo de contacto entre la fase liquida y sólida, conllevando esto a un menor grado de conversión de la materia orgánica afluente. Es así como para la condición correspondiente a un tiempo de retención de 6.9 h, la remoción de este parámetro solo alcanza el $39 \%$.

El humedal entre tanto, contribuyó a pulir el efluente del FAa pesar de que la remoción de materia orgánica es menor, esto se puede explicar por el bajo tiempo de retención hidráulico con que trabajó el sistema y la baja carga orgánica aplicada al mismo. Esta condición en el humedal fue igual para los dos sistemas, mostrando que esta unidad cuando opera a valores pequeños de TRH sufre reducciones considerables en la eficiencia de remoción de materia orgánica, lo cual confirma lo reportado previamente en la literatura (Haberl et al., 2003).

En general se puede afirmar que el sistema de tratamiento de aguas residuales $\mathrm{TS}+\mathrm{FA}+\mathrm{HFS}$, en ambos estudios tanto a nivel piloto como a escala real fue flexible a los cambios de caudal. Lo anterior es importante en la definición de criterios de diseño, ya que al operar el sistema a mayores cargas se logra una reducción efectiva en el área necesaria de tratamiento, lo que se revierte en una disminución de los costos de inversión inicial representados principalmente en área de terreno y construcción del sistema. 
Con base en la normatividad Colombiana (decreto 1594/1984), se puede afirmar que el sistema integrado (TS+FA+HS) puede soportar variaciones del doble de la carga hidráulica de diseño y cumplir con las exigencias de la citada norma $(80 \%$ de remoción para $\mathrm{DBO}_{5}$ y SST).

\section{Remoción de Nutrientes y coliformes fecales}

En el sistema de Ginebra, la remoción de nutrientes (NTK y $\mathrm{P}_{-} \mathrm{PO}_{4}{ }^{2-}$ ) y de coliformes fecales se redujo a medida que el sistema fue sobrecargado, siendo más notorio en el humedal, tecnología que fue ubicada como unidad terciaria, y donde se encontraron bajas eficiencias de remoción para TRH de 6 horas, alcanzando valores de $15 \%$ para NTK, $15 \%$ para P$\mathrm{PO}_{4}^{2-}$, y $60 \%$ para coliformes fecales. Esta condición es similar a lo reportado en la literatura, donde en esta unidad, a bajos tiempos de retención hidráulico la eficiencia de remoción de nutrientes y coliformes fecales se reduce significativamente (Reed et al., 1995; Haberl et al., 2003).

Un aspecto importante respecto al sistema de tratamiento de Ginebra, es que el humedal reportó eficiencias de remoción bajas en las condiciones de sobre carga, situación que está asociada al corto tiempo de retención de esta unidad $(\mathrm{TRH}=12 \mathrm{~h})$, el cual acorde a lo reportado a la literatura es muy pequeño si se pretende remover nutrientes y patógenos (Haberl et al., 2003; Brix, 1994).

En cuanto a la unidad experimental de La Vorágine, cuando el sistema operó a un caudal equivalente al de diseño $\left(104 \mathrm{~m}^{3} / \mathrm{d}\right)$ la remoción de NTK fue del 48\%. Una observación que debe destacarse en éste sistema es que gran parte del NTK se removió en la unidad de tanque séptico (29\%), lo cual pudo estar asociado a fenómenos de sedimentación y a una probable amonificación por la hidrólisis de proteínas y posterior degradación de aminoácidos, todo ello favorecido por el alto tiempo de retención en esta unidad primaria ( $\mathrm{TRH}=24$ horas).

De igual manera, en el sistema de Ginebra, la remoción de NTK y $\mathrm{P}_{-} \mathrm{PO}_{4}^{2-}$ en el humedal no presentó una reducción significativa para ninguno de los caudales estudiados, a pesar que esta unidad se construyó con dicho propósito.
Un aspecto importante que puede incidir en la baja remoción de nutrientes en el HFS, es que gran parte del nitrógeno afluente a esta unidad está bajo la forma de $\mathrm{N}$-amoniacal, producto de la transformación del $\mathrm{N}$-orgánico en las unidades precedentes, y para la oxidación del amonio se requiere de una fuente de carbón y oxígeno, elementos estos que pueden ser bajos en el agua residual de la etapa terciaria y en las micorrizas de las plantas, debido a las altas eficiencias de remoción de materia orgánica de las unidades de TS y FA. Así mismo, una baja tasa de transferencia de oxigeno de la planta a la zona de raíces, puede contribuir a la poca oxidación de Namoniacal, lo cual ha sido reportado en la literatura como uno de los factores que limitan la pérdida de nitrógeno en HFS (Reed et al., 1995).

Respecto a la remoción de indicadores de contaminación fecal (coliformes fecales), para el caso de la Vorágine las eficiencias en las unidades anaerobias (TS + FA) fluctuaron para las condiciones experimentales entre 74 y $96 \%$, valores bajos y que están lejos de generar un efluente seguro para su posterior reuso, pero que están en el rango promedio de remoción reportado en la literatura para sistemas anaerobios (Van Haandel y Lettinga, 1994). La remoción de Coliformes fecales en todo el sistema, no varió significativamente.

En el caso de Ginebra, la situación es similar a la de la Vorágine, pero con remociones menores, fluctuando entre el 69 y $71 \%$ para todo el sistema y donde el humedal tampoco presentó buenas remociones.

Para el caso del Humedal en ambos sistemas, la baja remoción de indicadores de contaminación microbiológica, asociada con el incremento de caudal, puede ser debida a que los mecanismos de remoción, como son el atrapamiento físico, la sedimentación, la adsorción y la predación no se propician por los bajos tiempos de retención, las altas velocidades de flujo y eventualmente por los gradientes de concentración de nutrientes que estimulan el recrecimiento y/o mantenimiento de la flora microbial del sistema, situación ésta que concuerda con lo reportado en la literatura técnica (Reed et al., 1995). 


\section{Conclusiones}

$\square$ El sistema Integrado TS + FA + HFS, mostró ser una excelente combinación para la remoción de materia orgánica, y cumplió con la reglamentación Colombiana (decreto 1594 de 1984) para remoción de $\mathrm{DBO}_{5}$ y SST.

चEl sistema Integrado TS + FA + HFS, puede soportar variaciones de caudal hasta el doble de su condición de diseño manteniendo las eficiencias de remoción de materia orgánica sin detrimentos significativos.

$\square \mathrm{La}$ remoción de nutrientes y patógenos en el sistema HFS fue afectada por el incremento de caudal y los bajos tiempos de retención hidráulico, produciendo un efluente de baja calidad microbiológica para su uso posterior en la agricultura o piscicultura.

$\square$ Para los pequeños municipios y comunidades rurales, el sistema integrado TS + FA+ HFS es una excelente opción para el tratamiento de las aguas residuales ya que mantiene su capacidad de remoción de materia orgánica a pesar de sufrir cambios drásticos en variables operativas como la tasa hidráulica aplicada.

$\square$ La flexibilidad de operación encontrada en el sistema integrado por TS + FA + HFS, es muy importante para la definición de criterios de diseño ajustados a las condiciones locales, dado que a mayores cargas se necesitan menores áreas del sistema, lo que se traduce en bajos costos de inversión inicial.

\section{Agradecimientos}

Los autores expresan su agradecimiento al personal de ACUAVALLE S.A, E.S.P, a la Universidad del Valle y a los siguientes profesionales: Ana Lucia Jácome, Claudia Ximena Marín, Luz Ángela Otero, Maria Fernanda Marín y Grover Rivera.

\section{Referencias bibliográficas}

American Public Health Association, American Water Works Association, Water Environmental
Federation. (1992). Standard Methods for Examination of Water and Wastewater. 14 edn. Washington DC, USA.

Brix, H. (1994). Constructed Wetland for Municipal Treatment in Europe. University of Aarhus. Denmark.

CEPIS. (2000). Evaluación de los Servicios de Agua Potable y Saneamiento en las Américas.

Doelle, H.W. (1998) Socio-economic microbial process strategies for a sustainable development using enviromentally clean technologies. Renewable Resource: Sagopalm.Editors:Eng-Leong Foo \& Tarcisio Della Senta. http/www.ias.unu.edu/ proceedings/icbs.

Feachem, R. and Cairncross, S. (1993). Environmental health engineering in the tropics. John Wiley \& Sons. New York, USA.

Haberl, R.; Grego, S.; Langergraber, G; Kadlec, R.; Cicalini, A.; Martins Dias, S.; Novais, J.; Aubert, S.; Gerth, A.; Thomas, H. y Hebner, A. (2003). Constructed Wetlands for the Treatment of Organic Pollutants. Journal of Soils \& Sediments 3(2), 109124.

Metcalf and Eddy. (1995) Ingeniería de Aguas Residuales. Tratamiento, Vertido y reutilización. $3^{\text {a }}$ Edición, Madrid: McGraw-Hill.

Ministerio de Salud. (1984). Decreto 1594. Reglamentación de los vertimientos de los desechos líquidos. Santa fé de Bogotá. Colombia.

Moscoso, J; y Leon, G. (1994). Uso de Aguas Residuales. Hoja de Divulgación Tecnica. OPSCEPIS (59), Lima, Perú.

Reed, S.; Crites, R y Middlebrooks, E. (1995). Natural System for Wastewater Management and Treatment. New York: McGraw Hill.

Van Haandel, A y Lettinga, G. (1994). Anaerobic Sewage Treatment. A practical Guide for Regions with Hot Climate, John Wiley \& Sons Ltd. Chinchester, UK. 